\title{
A Comparison of Orthogonalized Plane Wave and Augmented Plane Wave Methods for Calculating Photodetachment Cross-Sections
}

\author{
MANIJEH MOHRAZ AND LAWRENCE L. LOHR, JR. \\ Department of Chemistry, University of Michigan, Ann Arbor, Michigan 48109, USA
}

\begin{abstract}
s
The orthogonalized plane wave (OPW) method of calculating electronic continuum wavefunctions is tested by the computation of photodetachment cross-sections and angular distributions for gaseous halide anions. The results are compared to those obtained by a related augmented plane wave (APW) method involving the exact solution of a single-particle Schrödinger equation containing a piecewise Coulombic potential energy. These comparisons, as well as other involving experimental and theoretical cross-sections from the literature, indicate that OPW cross-sections are, at best, only semi-quantitatively reliable for describing photodetachment even at low photon energies, and that OPW cross-sections should be calculated using the dipole length operator rather than the dipole velocity operator.

La méthode des ondes planes orthogonalisées (OPW) pour calculer des fonctions d'onde du spectre continu a été testé par un calcul des sections efficaces et des distributions angulaires associées à des processus de photodétachement pour des anions halogénoïdes. Les résultats sont comparés à ceux obtenus par la méthode des ondes planes augmentées (APW), qui comporte la solution exacte d'une équation de Schrödinger à une particule, avec un potentiel coulombien par morceaux. Ces comparaisons ainsi que d'autres basées sur des sections efficaces expérimentales et théoriques de la littérature indiquent que les sections efficaces OPW ne sont guerre dignes de confiance même pour des énergies photoniques basses, et qu'elles doivent être calculées avec l'opérateur dipolaire de longueur plutôt qu'avec celui de vitesse.

Die OPW-Methode (orthogonalisierte ebene Wellen) für die Berechnung von elektronischen Kontinuumfunktionen wird mit einer Berechnung der mit Photoauslösevorgängen zusammenhängenden Querschnitte und Winkelverteilungen für Halogenidanionen getestet. Die Ergebnisse werden mit denen verglichen, die durch eine APW-Methode erhalten werden. In dieser Methode wird eine exakte Lösung einer Schrödingergleichung für ein Teilchen mit einem stückweise coulombischen Potential erhalten. Diese Vergleichungen als auch andere, basierend auf experimentelle und theoretische Literaturquerschnitte, deuten an dass die OPW-Querschnitte nicht einmal für geringe Photonenenergien zuverlässig sind, und dass sie mit dem Dipollänge-operator eher als mit dem Dipolgeschwindigkeitsoperator berechnet werden sollen.
\end{abstract}

\section{Introduction}

The rapidly increasing use of photoelectron spectroscopy has stimulated much recent interest in the calculation not only of ionization potentials but also of photoionization cross-sections and angular distributions. The desire to treat theoretically molecules of chemical interest has led to many studies using either plane waves (PW's) or orthogonalized plane waves (OPW's) to represent the ejected electrons [1-11]. The use of OPW continuum functions may be viewed [4]

(C) 1976 by John Wiley \& Sons, Inc. 
as the use of plane wave (PW) continuum functions together with considerations of the nonorthogonality of PW's to core orbitals and of the requirement of total wave-function antisymmetry. The results obtained to date by this method for a variety of small molecules appear to be semi-quantitatively reliable and very useful to photoelectron spectroscopists in making assignments. Particularly valuable have been the studies $[10,11]$ showing how the variation with photon energy of the cross section for ionization from a given molecular orbital is related to the symmetry and composition of that orbital. However, serious misgivings have frequently been raised [12] about even the approximate validity of the OPW method. It is the object of this paper to explore the relationship of the OPW method to more nearly exact methods of calculating continuum wave-functions and to present numerical results for atomic photodetachment cross-sections and angular distributions.

We have chosen to study photodetachment of anions rather than photoionization of neutrals because the continuum wave-functions in the former case are phase-shifted plane waves, which are more closely related to OPW's than are the phase-shifted Coulomb waves appropriate to the latter case. The specific systems studied are the gaseous halide ions, for which there have been numerous experimental and theoretical investigations of photodetachment cross-sections. Since these studies are largely confined to an energy range not exceeding $1 \mathrm{Ry}(13.6 \mathrm{eV})$ above the detachment threshold, our studies are made in the range from zero to $10 \mathrm{Ry}$ to facilitate comparisons. Thus, we are not considering in detail the high energy range for which the PW method and its derivative, the OPW method, are presumed to be most nearly valid. The compensating factor, as mentioned above, is the choice of anions rather than neutrals to study, thus eliminating the need to consider a long range Coulomb interaction. However, the calculation of cross sections for $p$-electron detachment, as in the case of the halides, is a demanding test since there are two final states for the electron, an $s$-state with a comparatively large phase shift and a $d$-state with a comparatively small phase shift. This study serves as a model for the detachment of non-bonding electrons from such species as $\mathrm{OH}^{-}, \mathrm{SH}^{-}, \mathrm{SeH}^{-}, \mathrm{NH}_{2}^{-}, \mathrm{PH}_{2}^{-}$, and $\mathrm{AsH}_{2}^{-}$, each isoelectronic with a halide ion and each studied by either crossed-beam [13] or ion cyclotron resonance [14] techniques. Earlier PW calculations of photodetachment cross sections include the studies of Bates and Massey [15] and Chandraskhar [16] on $\mathrm{H}^{-}$, and those of Moskvin $[17,18]$ on $\mathrm{Li}^{-}, \mathrm{Na}^{-}, \mathrm{K}^{-}, \mathrm{C}^{-}, \mathrm{N}^{-}, \mathrm{O}^{-}, \mathrm{F}^{-}$, and $\mathrm{Cl}^{-}$. A recent study by Reed et al. [19] reports cross-sections for $\mathrm{H}^{-}, \mathrm{C}^{-}, \mathrm{O}^{-}, \mathrm{F}^{-}, \mathrm{OH}^{-}, \mathrm{O}_{2}^{-}, \mathrm{CN}^{-}, \mathrm{C}_{2}^{-}$, and $\mathrm{C}_{5} \mathrm{H}_{5}$ calculated using $\mathrm{PW}$ 's orthogonalized only to the orbital from which detachment occurs. Other theoretical methods are described at the end of the next section.

\section{Theoretical Methods}

The differential cross-section for producing photoelectrons in the solid angle $d \Omega$ is given $[1,3,9]$ in the dipole approximation by the expression

$$
\frac{d \sigma}{d \Omega}=\frac{\pi e^{2}}{m^{2} c \omega}\left|\mathbf{u} \cdot\left\langle a\left|\sum_{n} \mathbf{p}_{n}\right| b\right\rangle\right|^{2} \rho(E)
$$


where $e$ is the magnitude of the electron charge, $m$ the electron mass, $c$ the velocity of light, $\omega$ the circular frequency, $\mathbf{u}$ a unit vector in the direction of the electric polarization, $a$ and $b$ the initial and final states respectively, $\mathbf{p}_{n}$ the linear momentum operator $-i \hbar \nabla_{n}$ for the $n$th electron, and $\rho(E)$ the density of final states with an electron kinetic energy $E=\hbar \omega-B E$, where the binding energy BE for photodetachment equals the electron affinity EA. If the initial state $\langle a|$ is a determinant of doubly occupied atomic orbitals and the final state $|b\rangle$ is a spin singlet constructed by promoting one electron from the $j$ th atomic orbital to an unbound orbital $|k\rangle$, with no change in the shape of the other atomic orbitals, then

$$
\left\langle a\left|\sum_{n} \mathbf{p}_{n}\right| b\right\rangle=2^{1 / 2}\langle j|\mathbf{p}| k\rangle
$$

where the factor $2^{1 / 2}$ accounts for the fact that there are two electrons in orbital $j$. The matrix element of the linear momentum operator $\mathbf{p}$ in Eq. (2) may be written in terms of dipole length operator $\mathbf{r}$ as

$$
\langle j|\mathbf{p}| k\rangle=\frac{-\mathrm{im}\left(E_{k}-E_{j}\right)}{\hbar}\langle j|\mathbf{r}| k\rangle
$$

where $E_{k}-E_{j}$ corresponds to the photon energy $\hbar \omega$.

In this study each bound atomic orbital $\langle j|$ is taken to be a superposition of Slater-type basis functions. The orbitals used are those obtained by selfconsistent-field (SCF) calculations on the atomic halides [20-23].

Two types of continuum state functions $|k\rangle$ are used in our photodetachment cross section, namely orthogonalized plane waves (OPW's) and wave-functions derived from a central-field model. The latter type of function is related to a plane wave which is phase-shifted at distances far from the nucleus and which is augmented by Coulomb-type functions at distances close to the nucleus. Therefore it will be referred to as an augmented plane wave (APW).

The adaptation of Eq. (1) to OPW final states has been given by several authors $[4,9]$ and will be only briefly described. A box-normalized plane wave (PW)

$$
|k\rangle=|\mathrm{PW}(\mathbf{k})\rangle=L^{-3 / 2} e^{i \mathbf{k} \cdot \mathbf{r}}
$$

where $L$ is the box length, has a corresponding density of states

$$
\rho(E)=\frac{m k L^{3}}{2 \pi^{2} \hbar^{2}}
$$

so that Eq. (1) reduces to

$$
\frac{d \sigma_{j}}{d \Omega}=\frac{e^{2} k L^{3}}{\pi m c \hbar^{2} \omega}|\mathbf{u} \cdot\langle j|\mathbf{p}| \mathrm{PW}(\mathbf{k})\rangle|^{2}
$$

where the subscript $j$ on $d \sigma / d \Omega$ denotes the atomic orbital from which detachment occurs. The magnitude of $k$ in Eqs. (5) and (6) is given by

$$
k=\left[\frac{2 m}{\hbar^{2}}(\hbar \omega-\mathrm{EA} j)\right]^{1 / 2}
$$


where $\mathrm{EA} j$ is the electron affinity for the $j$ th orbital. Since the PW is an eigenfunction of the linear momentum $\mathbf{p}$ with eigenvalue $\hbar \mathbf{k}$,

$$
\frac{d \sigma_{j}}{d \Omega}=\frac{e^{2} k L^{3}}{\pi m c \omega}(\mathbf{u} \cdot \mathbf{k})^{2}|\langle j \mid \mathrm{PW}(\mathbf{k})\rangle|^{2}
$$

Using the dipole length formulation,

$$
\frac{d \sigma_{j}}{d \Omega}=\frac{e^{2} k L^{3} m \omega}{\pi c \hbar^{2}}|\mathbf{u} \cdot\langle j|\mathbf{r}| \mathrm{PW}(\mathbf{k})\rangle|^{2}
$$

The integrals in the two expressions are quite different. In Eq. $(8)\langle j \mid \mathrm{PW}(k)\rangle$ is the overlap of the initial bound state with the PW, or simply the Fourier transform of the bound state into momentum space. In Eq. (9) the integral $\langle j|\mathbf{r}| \mathrm{PW}(k)\rangle$ corresponds to a transition moment from the initial states $\langle j|$ to the final state $|\mathrm{PW}(k)\rangle$. In the spirt of Eq. (8) one could write the matrix element in Eq. (9) as $\langle\mathbf{r} j \mid \mathrm{PW}(k)\rangle$, which is the overlap of a state $\langle\mathbf{r} j|$ with the PW.

Schmidt orthogonalization of the PW to the bound state yields

$$
|\mathrm{OPW}(\mathbf{k})\rangle=N\left[|\mathrm{PW}(\mathbf{k})\rangle-\sum_{l}\langle l \mid \mathrm{PW}(\mathbf{k})\rangle|l\rangle\right]
$$

where the sum is over all occupied orbitals $|l\rangle$. The normalization constant $N$ is given by

$$
N=\left[1-\sum_{l}\langle l \mid \mathrm{PW}\rangle\langle\mathrm{PW} \mid l\rangle\right]^{-1 / 2}
$$

As the size of the box within which the PW's are normalized becomes infinite, the overlap $\langle l \mid \mathrm{PW}\rangle$ becomes negligible compared to unity and $N$ approaches unity. Substituting Eq. (10) in Eq. (6),

$$
\frac{d \sigma_{j}}{d \Omega}=\frac{e^{2} k L^{3}}{\pi m c \hbar^{2} \omega}\left|(\mathbf{u} \cdot \hbar \mathbf{k})\langle j \mid \mathrm{PW}(\mathbf{k})\rangle-\mathbf{u} \cdot \sum_{1}\langle\mathrm{j}|\mathbf{p}| l\rangle\langle l \mid \mathrm{PW}(\mathbf{k})\rangle\right|^{2}
$$

The use of OPW's introduces into the cross-section expression bound-to-bound dipole matrix elements of the form $\langle j|\mathbf{p}| l\rangle$ multiplied by monopole matrix elements $\langle l \mid \mathrm{PW}(\mathbf{k})\rangle$.

The differential cross-sections are calculated for two fixed orientations of the polarization $\mathbf{u}$ with respect to the electron momentum $\hbar \mathbf{k}$, namely for the polarization $\mathbf{u}$ perpendicular to $\hbar \mathbf{k}$ and for $u$ parallel to $\hbar \mathbf{k}$. For detachment from orbitals other than $s$ orbitals an averaging procedure is required and may be carried out in either of two ways: a) by specifying the type of the orbital (for example $p_{x}, p_{y}$, or $p_{z}$ in the case of detachment from a $p$ orbital) and then averaging over the orientation of its axes with respect to the laboratory axes; or b) by calculating a simple average of the expressions for detachment from all the different orbitals in a given subshell for a fixed direction of $\mathbf{u}$ relative to $\mathbf{k}$.

In summary, the integrals that have to be evaluated in the OPW procedure are of three main types: overlap integrals $\langle j \mid \mathrm{PW}\rangle$ of atomic wave-functions with a $\mathrm{PW}$, 
bound-to-bound transition integrals $\langle j|\mathbf{r}| l\rangle$ and $\langle j|\mathbf{p}| l\rangle$, and dipole transition integrals of the form $\langle j|\mathbf{r}| \mathrm{PW}\rangle$.

In the second, or APW, method the continuum state wave-function is obtained by solving the radial Schrödinger equation

$$
\left[\frac{\hbar^{2} d^{2}}{2 m d r^{2}}-V_{n l^{\prime}}(r)+E-\frac{\hbar^{2} l(l+1)}{2 m r^{2}}\right] P_{E l}(r)=0
$$

where $l$ is the angular quantum number of the photoelectron, $V_{n l^{\prime}}(r)$ (which is designated with the subscript $n l^{\prime}$ to indicate that detachment is from the $n l^{\prime}$ th subshell) is an effective potential energy, and $P_{E l}(r)=r R_{E l}(r)$ is the continuum radial function. We have chosen to use a modification of the procedure developed by McGuire [24] and extended by Chapman and Lohr [25] for the solution of Eq. (13). In this procedure $V(r)$ is approximated by a model potential energy for which an exact solution of the radial equation can be determined. The potential energy corresponds to an electron in the field of a positive charge $Z e$ at the origin surrounded by a series of concentric shells at $R_{i}$, each having a uniform distribution of negative charge $-Z_{i} e$. The potential energy is piecewise Coulombic in all regions except the outermost, and hence solutions can be expressed in terms of Whittaker functions. These functions of the dimensionless variable $k r$ are evaluated in terms of their power series expansions for $k r \leq 11$ and with asymptotic formulas for $k r>11$. They involve a number of infinite but convergent series which are evaluated by approximating them as finite sums.

For the outermost region where $V_{n}(r)$ is zero, the solutions are spherical Bessel and Neumann functions. It is the form of the solutions in this outermost region that distinguishes the procedure from that used by McGuire [24] and by Chapman and Lohr [25] for describing the photoionization of neutral atoms. For each region of potential a general solution is constructed from the two particular solutions required to form the general solution of the second-order differential equation (13). The coefficients are evaluated by the application of suitable boundary conditions. A solution for the entire range of potential is then determined by insuring that the wave-function and its derivative are continuous at each boundary of the potential and that the wave-function has an appropriate form at limits of small and large $r$. The limiting boundary conditions for the continuum wave-function at small and large $r$ are

$$
P_{E l}=r R_{E l}(r) \rightarrow 0 \quad \text { as } r \rightarrow 0
$$

and

$$
P_{E l}(r)=r R_{E l}(r) \rightarrow k^{-1} \sin \left(k r-l \frac{\pi}{2}+\delta_{l}\right) \text { as } r \rightarrow \infty
$$

where $\delta_{l}$ is the phase shift of the $l$ th partial wave relative to a free wave (for which $V(r)=0$ for all $r$ ) due to the attractive potential of the residual atom. The phase shift is related to coefficients $A$ and $B$ for the outermost ( $n$ th) region by

$$
\tan \delta=-B / A
$$


The phase shift is assigned a positive sign to indicate that the potential is attractive. Since the spherical Bessel functions of order $l$ which occur in the outermost region may be viewed as spherical projections of a plane wave, the total continuum wave may be viewed as the $l$ th component of a phase-shifted plane wave suitably augmented by Coulomb waves in the inner regions, and hence its designation as an "augmented plane wave" (APW).

In order to characterize the piecewise potential energy $V(r)$, the set of parameters $R_{i}$ and $Z_{i}$ must be specified. This is done by fitting [25] the piecewise potential energy to a reference potential energy $V_{n l}(r)$, which is obtained from $\mathrm{SCF}$ orbitals for the core atom and which has the form

$$
V_{n l^{\prime}}(r)=\frac{-Z e^{2}}{r}+\frac{e^{2}}{r} \int_{0}^{r} \rho\left(r^{\prime}\right) d r^{\prime}+e^{2} \int_{r}^{\infty} \frac{\rho\left(r^{\prime}\right)}{r^{\prime}} d r^{\prime}
$$

in which

$$
\rho\left(r^{\prime}\right)=\sum_{\mu \lambda} \omega_{\mu \lambda} P_{\mu \lambda}^{2}\left(r^{\prime}\right)
$$

The summation is over the $n-1$ electrons in the residual atom. The occupation number $\omega_{\mu \lambda}$ for the $\mu \lambda$ th orbital includes electrons of both spins, but the exchange interaction between the residual atom and the continuum electron is not included explicitly. Note that the summation in Eq. (17) does not include the electron which is being ionized, so that the so-called "self-Coulomb energies" are absent. Parameters for the model potential energy are then determined by fitting $r$ times the reference potential energy of Eq. (16) with a series of line segments. The charge at the origin is taken as the true nuclear charge $Z e$, and the sum of the shell charges is $-Z e$. Thus, the model potential energy approches the correct asymptotic limit of zero. Since the fitting requires that the model and reference potential energies be equal at each of the boundaries, the model potential energy is more negative than the reference potential energy between boundaries. Exceptions to this occur for radii greater than or just slightly less than the radius of the outermost sphere.

If the bound-state orbitals in Eq. (17) are taken as those for the negative ion before detachment, then the detachment is assumed to be electronically vertical. This specifically neglects effects due to the relaxation of the core atom. The reference potential energy can also be calculated using bound-state orbitals of the residual neutral atom, in which case the potential energy corresponds to the relaxed core.

In an attempt to account for the polarization of the core, a polarization term may be added to the reference potential energy of Eq. (16). This term is taken as

$$
V_{p}(r)=-\frac{\alpha e^{2}}{2\left(r^{2}+r_{p}^{2}\right)^{2}}
$$

where $\alpha$ is the polarizability and $r_{p}$ corresponds roughly to the atomic core radius. It should be noted that the polarization term of Eq. (18) is not included explicitly in the model potential. The continuum wave-functions for all $n-1$ regions are still calculated for a piecewise Coulombic potential energy. The only effect of polari- 
zation is to make the reference potential energy to which the model potential energy is fitted more attractive.

The angular distribution of ejected electrons is characterized by an asymmetry parameter $\beta$ which appears in the differential cross-section [26] for polarized light as

$$
\frac{d \sigma}{d \Omega}=\frac{\sigma}{4 \pi}\left[1+\beta P_{2}(\cos \theta)\right]
$$

where $\theta$ is the angle between $\mathbf{k}$ and $\mathbf{u}$ and $P_{2}(x)=\left(3 x^{2}-1\right) / 2$. The total subshell cross-section $\sigma$ is the intensity parameter reported in Section 3. In the OPW method $\beta$ is conveniently obtained from

$$
\beta=2\left(\sigma_{\|}-\sigma_{\perp}\right) /\left(\sigma_{\|}+2 \sigma_{\perp}\right)=8 \pi\left(\sigma_{\|}-\sigma_{\perp}\right) / 3 \sigma
$$

where $\sigma_{\|}$and $\sigma_{\perp}$ denote the values of the differential cross-section for $\mathbf{k} \| \mathbf{u}$ and $\mathbf{k} \perp \mathbf{u}$, respectively. In the APW method, as with other central field methods, $\beta$ for $p$ electron detachment or ionization is given by the Cooper-Zare [26] relationship

$$
\beta=\frac{6 R_{d}^{2}-12 R_{d} R_{s} \cos \Phi}{3\left[R_{s}^{2}+2 R_{d}^{2}\right]}
$$

where $R_{s}$ and $R_{d}$ are the radial transition moments to the $s$ and $d$ final states and where $\Phi$ is the difference between the $s$ and $d$ phase shifts.

Other theoretical methods have been used for calculating photo-detachment cross-sections. Several of these are described below to show their relationship to the present work. The main difference among the methods is in the type of the bound and continuum state wave-functions that are employed and the manner in which they are obtained. The notations $P_{n l}(r)$ and $P_{E l}(r)$ are used to designate the bound and the continuum radial wave-function, $r R_{n l}(r)$ and $r \dot{R}_{E l}(r)$, respectively.

Cooper and Martin [27] calculated photodetachment cross-sections for $\mathrm{O}^{-}$, $\mathrm{C}^{-}, \mathrm{Cl}^{-}$, and $\mathrm{F}^{-}$, making the assumption that $P_{n l}(r)$ and $P_{E l}(r)$ are eigenfunctions of the same one-electron Hamiltonian having a potential energy

$$
V^{\prime}(r)=-\frac{Z e^{2}}{r}+\frac{d r^{\prime} \rho\left(r^{\prime}\right) e^{2}}{\left|r-r^{\prime}\right|}-\frac{\alpha e^{2}}{2\left(r^{2}+r_{p}^{2}\right)^{2}}
$$

where the first two terms correspond to the electrostatic potential energy. The factor $\rho\left(r^{\prime}\right)$ is the electronic charge distribution derived from the available Hartree or Hartree-Fock wave-functions. The correction term (with $\alpha$ ) is allowed to absorb the effects of polarization and exchange as they effect the binding energy $E_{b}$, which is taken as an experimental electron affinity. The factor $r_{p}$ is taken to be the average core radius. The bound function $P_{n l}(r)$ is then obtained treating $\alpha$ as an eigenvalue. Using the calculated value of $\alpha$, the continuum-state wavefunctions $P_{E l}(r)$ are then evaluated for various values of electron energy.

The chief advantages of this procedure are that it yields bound radial orbitals with correct binding energies and that it includes polarization effects, with the potential energy approaching the correct form $-\alpha e^{2} / 2 r^{4}$ for large $r$. A major 
shortcoming is that the semi-emperical parameters $\alpha$ and $r_{p}$ do not arise naturally from the formalism and are introduced somewhat arbitrarily.

Using a related procedure, Robinson and Geltman [28] calculated photodetachment cross-sections for a number of atomic negative ions. The potential energy which they used has the form

$$
V^{\prime}(r)=V_{\mathrm{HS}}(r)+\frac{e^{2}}{r}\left(1-e^{-r / r_{0}}\right)-\frac{\alpha e^{2}}{2} \frac{\left(1-e^{-r / r_{p}}\right)}{\left(r^{2}+r_{p}^{2}\right)^{2}}
$$

where $V_{\mathrm{HS}}(r)$ is the Hartree-Fock-Slater potential energy for the neutral atoms, evaluated by the methods of Herman and Skillman [29]. The second term removes the Coulomb tail of $V_{\mathrm{Hs}}$ and the third term introduces the effects of polarization. As in the previous case, the parameter $r_{p}$ is chosen somewhat arbitrarily to correspond to core radius. The atomic polarizability $\alpha$ is chosen from the best existing theoretical or experimental values, while the parameter $r_{0}$ is chosen such that the resulting potential will support an $n p$ bound state with an assumed binding energy. The bound- and continuum-state wave-functions are then calculated in a manner similar to the method of Cooper and Martin.

Garrett and Jackson [30] used a more sophisticated procedure to calculate photodetachment cross sections of $\mathrm{O}^{-}$. The bound-state wave-functions $\boldsymbol{P}_{n l}(r)$ are calculated by the modification of the Hartree-Fock-Slater (HFS) method, in which the potential energy is the sum of the electrostatic energy and an exchange energy $V_{\mathrm{ex}}(r)$ given in terms of the electron density $\rho(r)$ by

$$
V_{\mathrm{ex}}(r)=-3 e^{2} \lambda\left[\frac{3}{8 \pi} \rho(r)\right]^{1 / 3}
$$

where the coefficient $\lambda$ is varied until an eigenvalue of the $2 p$ electron is obtained which corresponds to the experimental binding energy of $\mathrm{O}^{-}$. This method of the calculation of the bound-state wave-functions is different from that used by Cooper and Martin or Robinson and Geltman in that a self-consistent calculation is made each time the parameter $\lambda$ is adjusted rather than taking a single equation and adjusting a parameter in the total potential function (the parameter $r_{p}$ in the method of Cooper and Martin or the parameter $r_{0}$ in the method of Robinson and Geltman) in order to match the desired eigenvalue. The continuum functions are then obtained by numerical integration of the radial Schrödinger equation containing a polarization potential obtained from an application of first-order perturbation theory to the Hartree-Fock (HF) atomic system. The method leads to substantial improvement in the agreement of theory and experiment over the methods of Cooper and Martin or Robinson and Geltman. The major difference in their results for $\mathrm{O}^{-}$as compared to those of the previous methods stems from differences in the continuum functions which are very sensitive to small differences in the polarization potential.

A somewhat different approach is the close-coupling method used by Conneely, Smith, and Lipsky [31] for electron impact and photoionization as well as 
for photodetachment. In lieu of a polarization potential their method explicitly includes low-lying excited terms in the final-state wave-functions, although again SCF initial-state functions are chosen. Very recently, Ishihara and Foster [32] studied the photodetachment of $\mathrm{C}^{-}$and $\mathrm{F}^{-}$using many-body perturbation theory in which they included the effects due to correlation and to intrachannel interactions. The calculated cross-sections are in very good agreement with the experimental results, showing the importance of the above corrections in the crosssection calculations.

\section{Results and Discussions}

\section{A. General Remarks}

The photodetachment cross-section $\sigma$ and the asymmetry parameter $\beta$ are calculated by the OPW and APW methods for the $n p$ photodetachment of halide ions. In all cases both the dipole length and dipole velocity formulations are employed. In order to facilitate comparisons the same SCF bound-state functions are used in the PW, OPW, and APW calculations. These functions enter the OPW calculations both as the initial-state functions $\langle j|$ and as the functions to which a PW is Schmidt orthogonalized. They enter the APW calculation both as the initial-state functions $\langle j|$ and as the functions used to calculate the reference potential energy in Eq. (16). Since SCF functions for $\mathrm{X}^{-}$rather than $\mathrm{X}^{\circ}$ are used throughout, effects associated with core relaxation are ignored. The spin-orbit splitting of the $n p^{5}$ core of the $\mathrm{X}^{0}$ atom is ignored except in the series of APW calculations including core polarization, where the splitting is considered in order to facilitate comparisons of our results with other calculated values and with experimental values.

Table I is a list of the electron affinities for the halide ions. The values in the first column are one-electron energies [20-23] corresponding to the bound-state orbitals of $\mathrm{X}^{-}$. The values in the second column correspond to the difference in the neutral and negative ion total energies, where both energies are calculated [20-22] from wave-functions with comparable basis sets. The third column corresponds to the experimental electron affinities [33]. For most of the ions the

TABLE I. Electron affinities (eV).

\begin{tabular}{lccc}
\hline Atom & $\begin{array}{c}\text { one-Electron } \\
\text { Energies }\end{array}$ & $\begin{array}{c}\text { Difference of } \\
\text { Total Energies }\end{array}$ & Experimental \\
\hline $\mathrm{F}$ & $4.93^{\mathrm{a}}$ & 1.39 & 3.45 \\
$\mathrm{Cl}$ & $4.13^{\mathrm{b}}$ & 2.59 & 3.61 \\
$\mathrm{Br}$ & $3.77^{\mathrm{C}}$ & 2.58 & 3.36 \\
$\mathrm{I}$ & $3.10^{\mathrm{d}}$ & - & 3.06 \\
\hline${ }^{\mathrm{a}}$ Ref. [20]. & ${ }^{\mathrm{b}}$ Ref. [21]. & ${ }^{\mathrm{c}}$ Ref. [22]. ${ }^{\mathrm{d}}$ Ref. [23]. ${ }^{\mathrm{C}}$ Ref. [33].
\end{tabular}


three electron affinities listed in Table I are significantly different. This poses a problem in choosing among them. Since for negative ions the calculated energies are not usually very accurate, the experimental electron affinities provide a better description of the process. On the other hand, the theoretical one-electron energies correspond to the bound-state functions which are used to calculate the photodetachment cross-sections, and in that sense they might be more appropriate.

In the cases where the values are different, the choice of one or the other makes a significant difference in the relative magnitude of the cross-sections calculated by the dipole length and dipole velocity methods, especially at very low electron energies. This is easily illustrated if one looks at the two expressions

$$
\begin{aligned}
& \sigma_{\mathrm{L}} \sim(E+\mathrm{EA})\left|\mathrm{ME}_{\mathrm{L}}\right|^{2} \\
& \sigma_{\mathrm{V}} \sim \frac{1}{(E+\mathrm{EA})}\left|\mathrm{ME}_{\mathrm{V}}\right|^{2}
\end{aligned}
$$

where the subscripts $\mathrm{L}$ and $\mathrm{V}$ correspond to dipole length and dipole velocity formulations, respectively. EA is the absolute value of the electron affinity and $E$ the kinetic energy of the photoelectron. In Eq. (24) the cross-section is directly proportional to the photon energy $\hbar \omega=E+E A$, whereas in the formulation of Eq. (25) it is inversely proportional to this factor. For example, at energies close to threshold the cross-section $\sigma_{\mathrm{L}}$ for $\mathrm{F}^{-}$increases by about $40 \%$ if the one-electron energy is used, while the cross-section $\sigma_{\mathrm{V}}$ decreases by the same amount. Therefore, at low energies the calculated cross-sections are very sensitive to the value used for the electron affinity. For the calculations reported here the experimental electron affinities are used.

\section{B. A Comparison of $P W$ and $O P W$ Results}

The variation of the PW and OPW cross-sections with photoelectron energy for ions $\mathrm{F}^{-}, \mathrm{Cl}^{-}, \mathrm{Br}^{-}$, and $\mathrm{I}^{-}$are shown in Figures 1 through 4 , respectively. For $\mathrm{F}^{-}$ (Fig. 1) with the dipole length formulation, both the magnitude and the shape of the cross-section are significantly different for the PW and OPW results in the energy range from 0 to about $2 \mathrm{Ry}$. The two approach each other as one moves to higher energies. The same is also true for the dipole velocity formulation. There is a similar pattern for the other three ions (Figs. 2-4). There is a similar pattern for the other three ions (Figs. 2-4). The difference is even greater if one compares the results of the two formulations in each of the two PW and OPW methods. As mentioned earlier, one must be cautious when comparing the cross-sections calculated by the dipole length and dipole velocity formulations, since at low photoelectron energies the relative magnitude of the two is very sensitive to the choice of the electron affinity. Indeed, using the theoretical orbital energy gives a better agreement between the two formulations at electron energies below $0.05 \mathrm{Ry}$. However, as the electron energy increases, the use of a greater electron affinity makes the agreement worse as the cross-sections calculated with the 


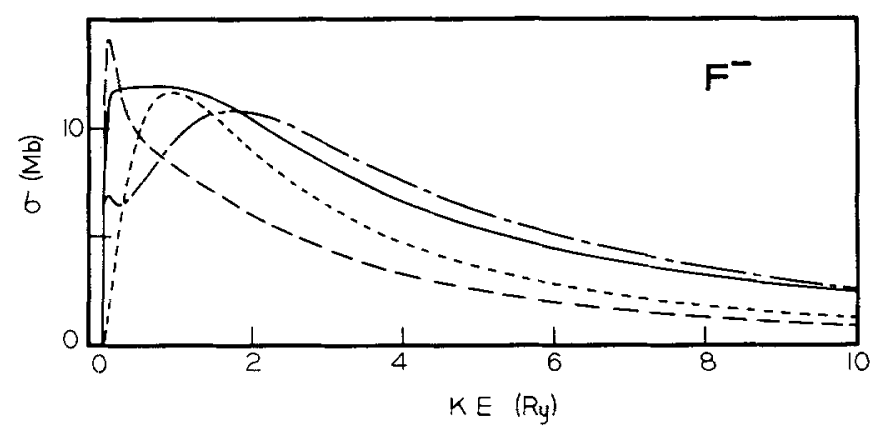

Figure 1. Cross-section $\sigma$ or in $\mathrm{Mb}$. vs. electron kinetic energy in Ry for $\mathrm{F}^{-}$. OPW (length) results denoted by $(-\cdots)$, PW (length) by (-), OPW (velocity) by (一 $(-$ ), and PW (velocity) by (- - - ). Curves in Figures 1-8 and 10-12 are drawn through points computed at intervals of $0.1 \mathrm{Ry}$ between zero and $1.0 \mathrm{Ry}$ and at intervals of 1.0 Ry above 1.0 Ry. Finer meshes were employed in some cases.

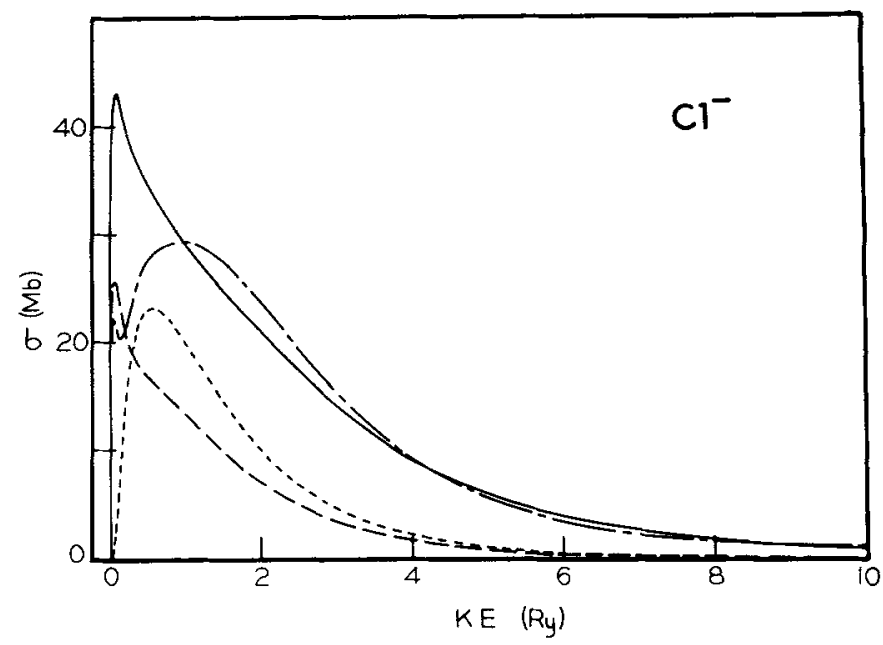

Figure 2. Cross section $\sigma$ in $\mathrm{Mb}$ vs. electron kinetic energy in $\mathrm{Ry}$ for $\mathrm{Cl}^{-}$. Curves labeled as in Figure 1.

dipole length formulation are becoming greater than those calculated with the dipole velocity operator. The "dips" in the plots of $\sigma$ at low electron energies are due to the fact that the cross-section for the photodetachment from a $p$ orbital is the sum of the cross-sections for the transition to the $s$ and $d$ continuum waves. For the $s$ transition the cross-section rises rapidly at very low electron energies, reaches a maximum, and then drops. For the $d$ transition the cross-section rises gradually with energy and reaches a maximum at much higher energies.

An important result that is not readily apparent in Figures 1-4 is that all of the curves except for those obtained using PW's and the velocity operator have the 


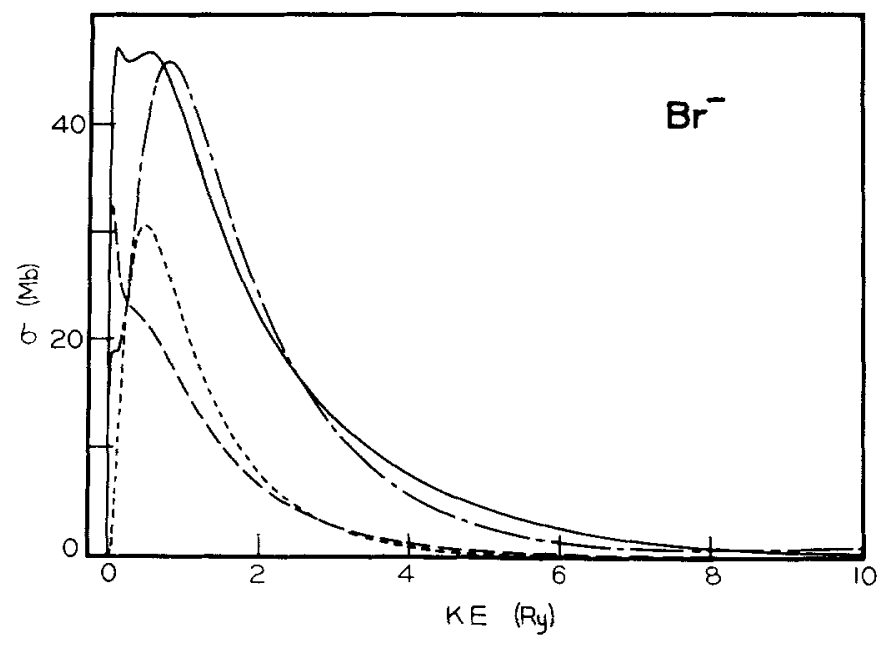

Figure 3. Cross-section $\sigma$ in $\mathrm{Mb}$ vs. electron kinetic energy in $\mathrm{Ry}$ for $\mathrm{Br}^{-}$. Curves labeled as in Figure 1.

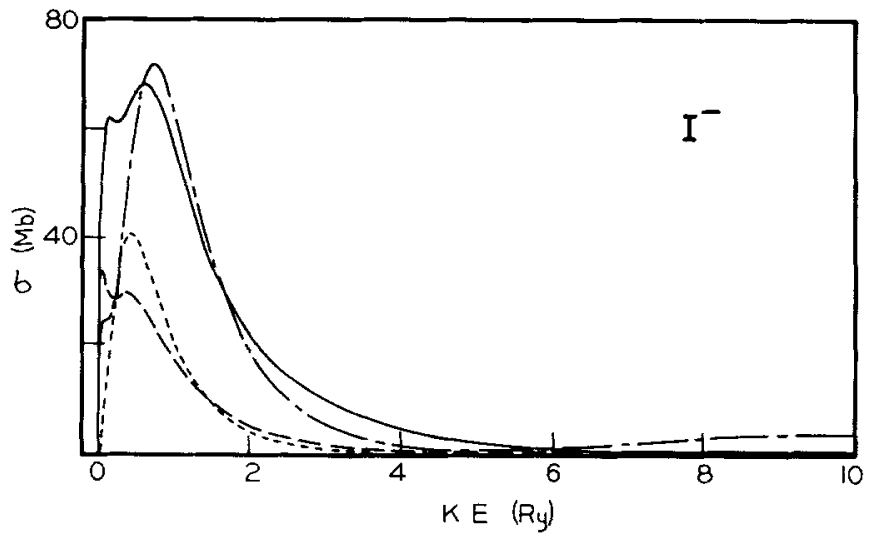

Figure 4. Cross-section $\sigma$ in Mb vs. electron kinetic energy in Ry for $\mathrm{I}^{-}$. Curves labeled as in Figure 1.

threshold behavior $\sigma \sim k$ expected from Wigner's rules [34] for the detachment of a $p$-electron. By contrast, the curves for the PW results with the velocity operator have the threshold form $\sigma \sim k^{3 / 2}$ for the detachment of any electron, a result that is only correct for $s$ - or $d$-electron detachment. The conclusion is that use of the length operator is necessary if PW's are used in order to have the correct threshold behavior. The same conclusion was reached by Reed et al. [19] in studies involving orthogonalization of the PW only to the orbital from which detachment occurs. However, the additional orthogonalization of the PW to $s$ orbitals builds into expression (14) the requisite final states for $\sigma$ so that either operator yields the 
correct threshold behavior. By contrast, at the higher energies shown in Figures 1-4 the $\sigma$ values are more dependent on the choice of operator than on orthogonalization, with the length values lying significantly above the velocity values for all halides.

Figures 5-8 show the variation of the PW and OPW values of the asymmetry parameter $\beta$ with photoelectron energy. The PW-dipole velocity formulation $\beta$ equals 2 at all electron energies and is not shown. For the other three methods, $\beta$ starts at zero, implying a spherical distribution at threshold. It drops to a minimum of -1 at about 0.1 to $0.2 \mathrm{Ry}$ and then rises to a value close to +2 . The shape of $\beta$ is approximately the same for all three methods. Also shown in these figures are

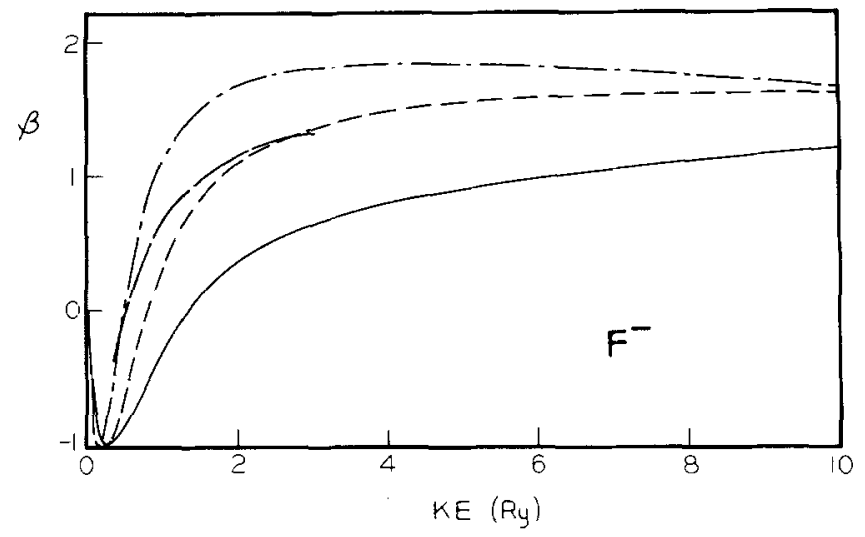

Figure 5. Asymmetry parameter $\beta$ vs. electron kinetic energy in Ry for $\mathrm{F}^{-}$. OPW

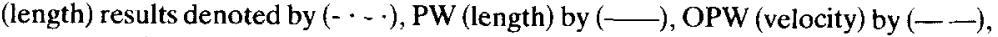
and APW (length) by (_ - - ). The PW (velocity) value of $\beta$ is 2 at all energies.

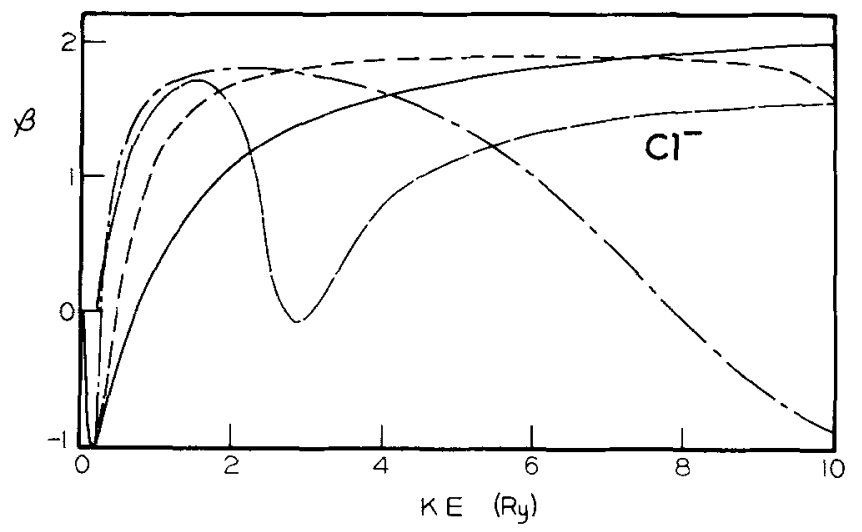

Figure 6. Asymmetry parameter $\beta$ vs. electron kinetic energy in $\mathrm{Ry}$ for $\mathrm{Cl}^{-}$. Curves labeled as in Figure 5. 


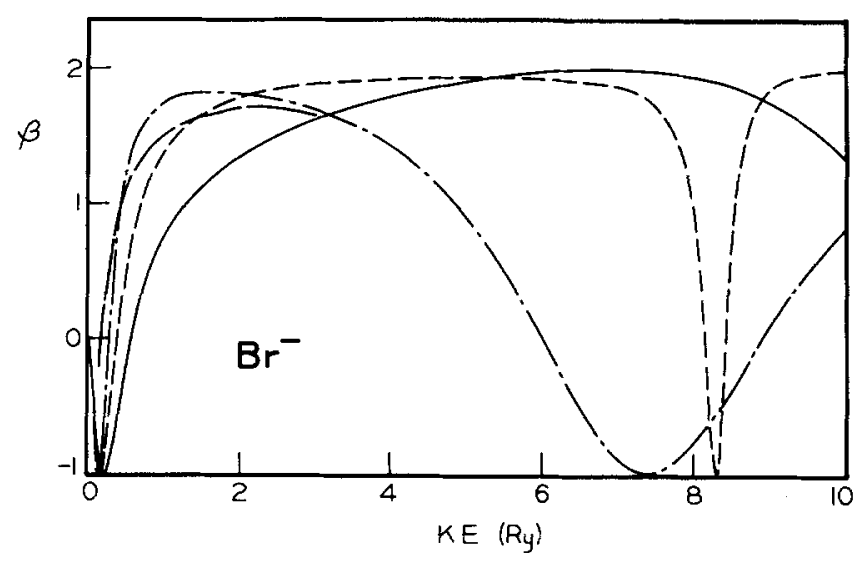

Figure 7. Asymmetry parameter in $\beta$ vs. electron kinetic energy in $\mathrm{Ry}$ for $\mathrm{Br}^{-}$. Curves labeled as in Figure 5.

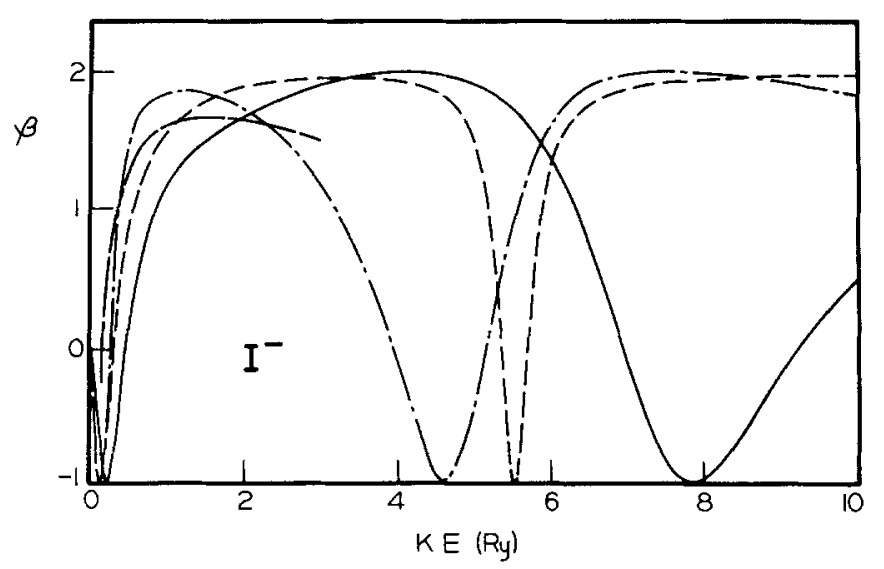

Figure 8 . Asymmetry parameter $\beta$ vs. electron kinetic energy in Ry for $\mathrm{I}^{-}$. Curves labeled as in Figure 5.

APW-dipole length $\beta$ values which are discussed in Section 3D. For $\mathrm{Cl}^{-}, \mathrm{Br}^{-}$, and $\mathrm{I}^{-}$, after reaching a maximum $\beta$ does not level off or decrease gradually, as for $\mathrm{F}^{-}$ (Fig. 5), but sharply drops to a minimum and then rises again. In the OPWdipole length method of Figure 6 for $\mathrm{Cl}^{-}$the second minimum occurs after $10 \mathrm{Ry}$. Going from $\mathrm{Cl}^{-}$to $\mathrm{I}^{-}$this minimum is shifted towards lower energies. For $\mathrm{Br}^{-}$it occurs at about 7.4 Ry and for I around 4.6 Ry. Although the OPW $\beta$ values are not calculated from Eq. (21), the equation may be used to analyze the results. Since the OPW phase shifts are zero, $\beta$ equals zero at energies for which $R_{d}=0$ or $R_{d}=2 R_{s}$; any relationship between the number of such crossings, and hence oscillations in $\beta$, to the number of bound-state radial nodes requires further exploration, although there is no relationship in the corresponding photoionization of $n p$ electrons from neutral rare gas atoms $[35,36]$. It has recently been 
stated [37] that since $\beta$ is so crucially dependent on the phase shifts there is no possibility, except by accident, of $\beta$ 's obtained by $P W$ methods, and presumably by OPW methods, being correct. While it would certainly be unjustified to attach other than semi-quantitative significance to the results in Figures 5-8, we do note that the curves have the correct threshold behavior for $p$-electron detachment as found theoretically by Cooper and Zare [38] using the Robinson-Geltman potential [28] and as found experimentally by Hall and Siegel [39]. Specifically, $\beta$ is zero at threshold, falls rapidly to a value near -1 , and then rises through zero to a value near 2 .

\section{A Comparison of APW and OPW Results}

In this section the APW cross-sections for the photodetachment from the $p$ orbital of halides are compared with the OPW results of the preceding section. Spin-orbit coupling is again neglected so that the cross-sections represent the sum over the ${ }^{2} P_{3 / 2}$ and ${ }^{2} P_{1 / 2}$ levels of the residual atom. Cross-sections were computed for all halides, but are listed for $\mathrm{F}^{-}$only in Table II with $E$ denoting the photoelectron energy and subscripts $L$ and $V$ the dipole length and dipole velocity formulations, respectively. The OPW results are the same as those shown in Figures 1-4.

TABLE II. Photodetachment cross-sections $(\mathrm{Mb})$ for $\mathrm{F}^{-}$.

\begin{tabular}{|c|c|c|c|c|c|}
\hline \multirow{2}{*}{$\lambda(\dot{A})^{a}$} & \multirow[b]{2}{*}{$E\left(R_{Y}\right)$} & \multicolumn{2}{|c|}{$A P W$} & \multicolumn{2}{|c|}{$O P W$} \\
\hline & & $\sigma_{L}$ & $\sigma_{\mathrm{v}}$ & $\sigma_{L}$ & ${ }^{\sigma} \mathrm{v}$ \\
\hline 3588 & 0.0 & 0.0 & 0.0 & 0.0 & 0.0 \\
\hline 2574 & 0.1 & 4.5 & 6.9 & 6.8 & 14.0 \\
\hline 2007 & 0.2 & 6.0 & 8.5 & 6.6 & 12.4 \\
\hline 1645 & 0.3 & 7.2 & 9.7 & 6.7 & 11.1 \\
\hline 1393 & 0.4 & 8.3 & 10.6 & 7.0 & 10.2 \\
\hline 1209 & 0.5 & 9.2 & 11.3 & 7.4 & 9.6 \\
\hline 1067 & 0.6 & 10.0 & 11.8 & 7.8 & 9.2 \\
\hline 955 & 0.7 & 10.8 & 12.1 & 8.3 & 8.9 \\
\hline 864 & 0.8 & 11.3 & 12.2 & 8.8 & 8.7 \\
\hline 727 & 1.0 & 12.3 & 12.2 & 9.6 & 8.2 \\
\hline 404 & 2.0 & 11.9 & 9.8 & 10.8 & 6.0 \\
\hline 214 & 4.0 & 6.5 & 5.2 & 7.6 & 3.3 \\
\hline 146 & 6.0 & 3.4 & 2.7 & 5.3 & 2.0 \\
\hline 110 & 8.0 & 1.9 & 1.5 & 3.7 & 1.3 \\
\hline 89 & 10.0 & 1.1 & 0.9 & 2.7 & 0.9 \\
\hline
\end{tabular}

${ }^{a}$ Using experimental $E A=0.254 \mathrm{Ry}$. 
The results of the dipole length and dipole velocity methods are generally different in magnitude. This may seem surprising since the APW continuum function represents an exact solution of the Schrödinger equation with the model potential energy $V_{M}(r)$. However, the SCF bound-state orbital is a solution of the Schrödinger equation with a different Hamiltonian, so that the identity in Eq. (3) does not hold. This point has been recently discussed by Starace [40] and by Cohen and McEachran [41]. In addition, we chose experimental rather than theoretical EA values, thus giving up any requirement of agreement of length and velocity cross sections. For $\mathrm{F}^{-}$(Table II) the agreement between the length and velocity formulations is fair, and in most cases the difference is less than $25 \%$. In both cases the maximum cross section occurs between 1.0 and $2.0 \mathrm{Ry}$.

For all the halides there is a general agreement in the shape of the $\mathrm{OPW}_{\mathrm{L}}$ cross-section and the position of its maximum with the APW results. However, the magnitude of the $\mathrm{OPW}_{\mathrm{L}}$ maximum cross-sections are generally lower than the corresponding APW values. The $\mathrm{OPW}_{\mathrm{V}}$ cross-sections are very different from the APW values and, as in the case of the length operator, the maximum OPW cross-section occurs at lower electron energies.

In Figure 9 we illustrate the PW, OPW, and APW $s$ continuum wave-functions for $\mathrm{F}^{-}$at an energy of $1.0 \mathrm{Ry}$. The functions are plotted as $P(r)=r R(r)$, with an

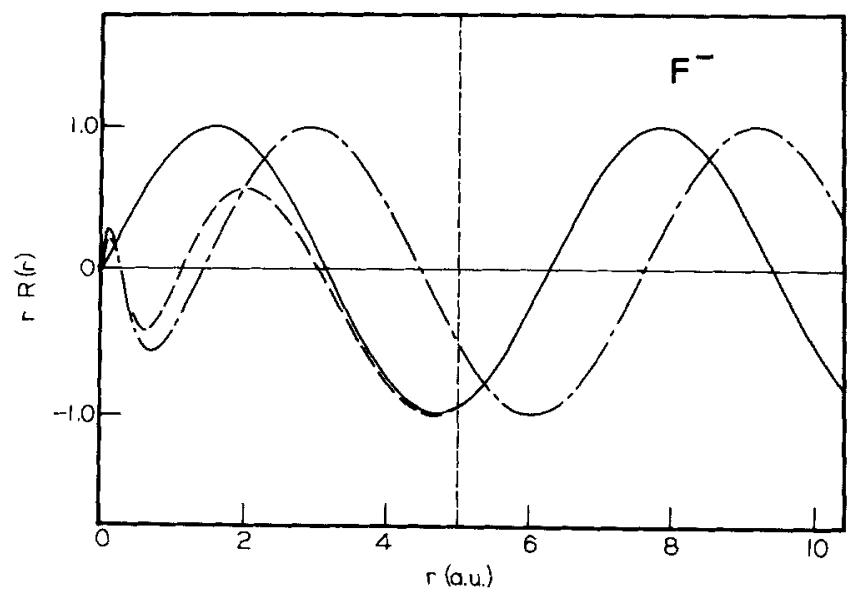

Figure 9. The $s$ continuum waves $r R(r)$ vs. $r$ in a.u. at an electron kinetic energy of 1.0 Ry for $\mathrm{F}^{-}$. APW denoted by (-- ), OPW by (- $)$, and PW by (- - ).

arbitrary unit amplitude so that the curve labeled PW is simply $k r j_{0}(k r)=\sin (k r)$, which is $k r$ times the $s$ component of the PW. The dashed vertical line at $r=5$ a.u. denotes the radius beyond which $V_{M}(r)$ is zero. We note that this is also the approximate radius beyond which the PW and OPW curves coincide. For $r<$ 5 a.u. the OPW function has one more maximum than does the PW function, but there is of course no long range phase shift. Another feature of the OPW function is that its nodes do not coincide with its points of inflection, so that there are 
singularities at the nodes in the local kinetic energy $T$ (local) $=(T \Psi) / \Psi$, where $T$ is the kinetic energy operator. Conversely, the OPW function may be viewed as an exact solution of a Hamiltonian containing a singular potential $E-T$ (local). By contrast, the APW function is an exact solution of a Hamiltonian containing a nonsingular potential, so we observe in Figure 9 that its nodes and points of inflection coincide. The $s$ phase shift at this energy is $1.57 \pi$, or approximately $3 \pi / 2$, so that the extrema at large $r$ nearly coincide with the nodes of the PW or OPW functions.

In Figure 10 we illustrate the PW and APW $d$ continuum functions for $\mathrm{F}^{-}$, again at an energy of $1.0 \mathrm{Ry}$. The PW function is $\operatorname{simply~} k r j_{2}(k r)$ and may be used

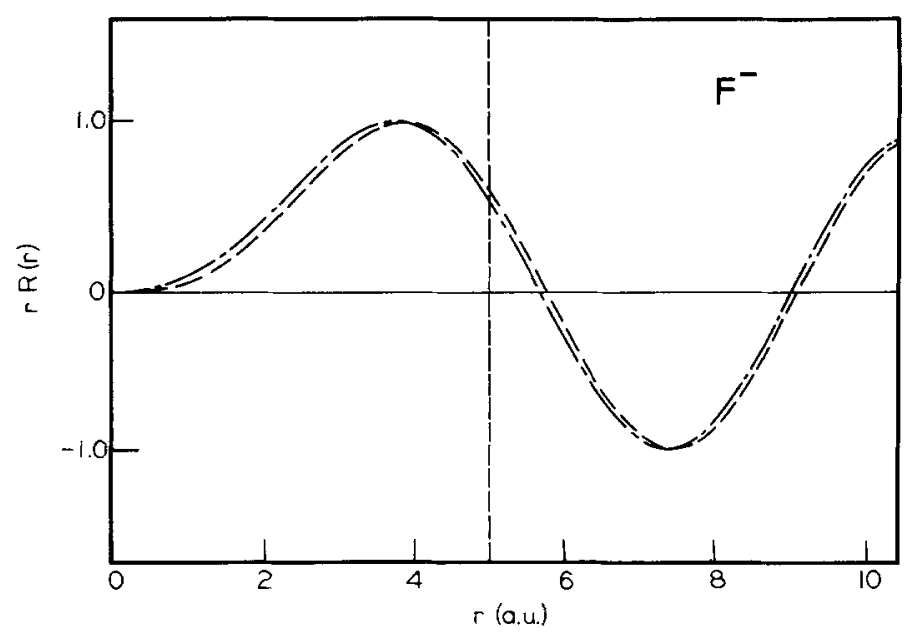

Figure 10. The $d$ continuum waves $r R(r)$ vs. $r$ in a.u. at an electron kinetic energy of 1.0 Ry for $\mathrm{F}^{-}$. APW denoted by (-- -) and OPW or PW denoted by (- -).

as an OPW function since $\mathrm{F}^{-}$has no occupied $d$ orbitals. As expected, the APW phase shift is quite small, namely $0.027 \pi$, so that the PW and APW functions are essentially identical at this energy.

\section{Inclusion of Core Polarization and Comparisons with Other Results}

APW continuum wave-functions are now evaluated for all halides using a reference potential energy which includes an empirical core polarization term $V_{\mathrm{p}}(r)$ of the form given in Eq. (18). The halogen polarizabilities are chosen either from theory [42] (F) or experiment [28] $(\mathrm{Cl}, \mathrm{Br}$, and I) are given in Table III together with values of the parameter $r_{p}$ chosen to correspond roughly to the atomic core radius. The effect of $V_{\mathrm{p}}(r)$ on the parameters defining the model potential energy $V_{\mathrm{M}}(r)$ is quite large. For $\mathrm{Cl}^{-}$the effect is to make $V_{\mathrm{M}}(r)$ at $r=3$ a.u. about twice as attractive as in the case with no $V_{\mathrm{p}}(r)$ in the reference potential energy. In addition, the region with $V_{M}(r)=0$ begins at $r=13.9$ a.u. when polarization is included in $V$, whereas the corresponding region begins at 
6.2 a.u. when polarization is not included. The polarization effects on $V_{\mathrm{M}}(r)$ are even greater for $\mathrm{Br}^{-}$and $\mathrm{I}^{-}$. As is well known from earlier studies [28], the inclusion of polarization increases the maximum value of the cross-section and moves its position to lower electron energies. For $\mathrm{Cl}^{-}$the $\mathrm{APW}_{\mathrm{L}}$ maximum without polarization is $46 \mathrm{Mb}$ at a kinetic energy of $0.7 \mathrm{Ry}$, while the $A P W_{L}$ maximum with polarization is $57 \mathrm{Mb}$ at $0.6 \mathrm{Ry}$.

Figure 11 compares the $A P W_{L}$ with polarization for $F^{-}$to the $O P W_{L}$ results, to the theoretical results of Cooper and Martin [27], Robinson and Geltman [28],

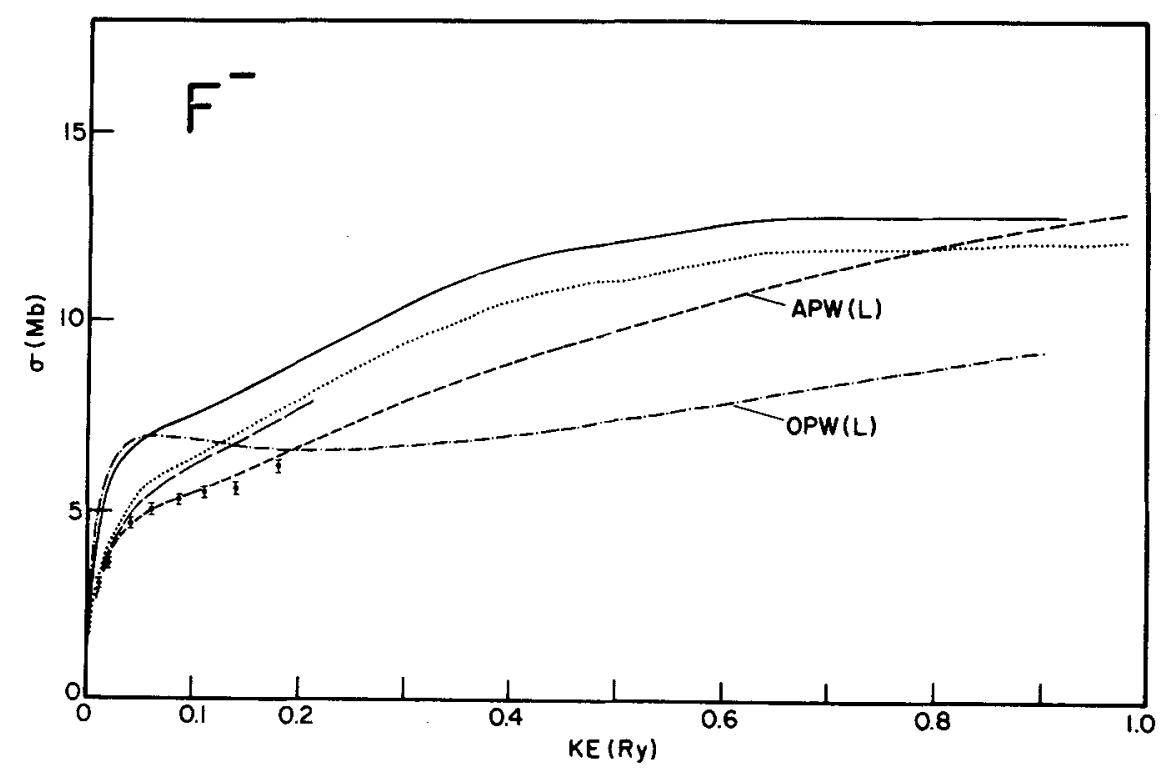

Figure 11. Cross-section $\sigma$ in $\mathrm{Mb}$ vs. electron kinetic energy in Ry for $\mathrm{F}^{-}$. APW (length) results with polarization denoted by (- -) and OPW (length) results by $(-\cdot)$. Other results are those of Cooper and Martin [27] denoted by $(\cdots)$, Robinson and Geltman [28] by (-), Ishihara and Foster [32] by (- - ), and Mandl $[43]$ by the points $\Phi$.

and Ishihara and Foster [32], and to the shock tube measurements of Mandl [43]. Except for electron energies below 0.2 Ry the $\mathrm{OPW}_{\mathrm{L}}$ values are much lower than the others. The excellent agreement between the APW $\mathrm{L}_{\mathrm{L}}$ calculations and Mandl's measurements, which have estimated uncertainties of $\pm 25 \%$, must in part be fortuitous, as our calculations ignore correlation in the initial state and both correlation and exchange in the final state. However, our reference potential energy used in calculating the APW final state does implicitly contain some correlation and exchange effects via the polarization term. Mandl [43] also compared his measurements to Robinson and Geltman's calculations, as well as to Moskvin's PW calculations [18] and to Berry and Reimann's [33] and Popp's [44] near threshold measurements. As Mandl's Figure 4 and our Figure 11 indicate, 
the Robinson-Geltman calculations agree reasonably well with experiment if their values are scaled by approximately 0.7 .

The experimental [45] spin-orbit splittings for the neutral halogens are given in Table III. We now include the effect of this splitting on the cross-sections for $\mathrm{Cl}$, $\mathrm{Br}$, and I by simply apportioning two-thirds of the computed cross-section to the lower $J=3 / 2$ level and one-third to the upper $J=1 / 2$ level, being careful to use $\sigma$ values computed for the desired kinetic energy, which for a given photon energy is different for the two levels, and to correct the photon frequency factors in Eqs. (24) and (25).

Figure 12 compares the $A P W_{L}$ results with polarization for $\mathrm{Cl}^{-}$to the $\mathrm{OPW}_{\mathrm{L}}$ and other theoretical results. The $\mathrm{APW}_{\mathrm{V}}$ values, not shown, are typically about

TABLE III. Polarization and spin-orbit parameters.

\begin{tabular}{lccc}
\hline Atom & $\left(a_{21}^{3}\right)^{\mathrm{N}}$ & $\mathrm{r}_{\mathrm{p}}(\mathrm{au})^{\mathrm{d}}$ & $\Delta \mathrm{F}_{\mathrm{so}}(\mathrm{Ry})^{\mathrm{C}}$ \\
\hline $\mathrm{F}$ & $4.05^{\mathrm{a}}$ & 1.5 & 0.0037 \\
$\mathrm{Cl}$ & $23.5^{\mathrm{b}}$ & 2.5 & 0.0080 \\
$\mathrm{Br}$ & $24.9^{\mathrm{b}}$ & 3.5 & 0.0336 \\
$\mathrm{I}$ & $40.5^{\mathrm{b}}$ & 4.5 & 0.0693 \\
\hline
\end{tabular}

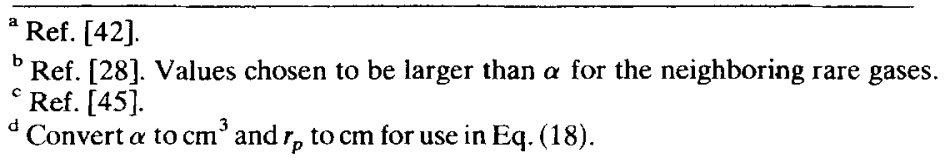

$1 / 3$ less than the $\mathrm{APW}_{\mathrm{L}}$ values over this energy range. The experimental $\sigma$ value of Berry and Reimann [33], Muck and Popp [46], and Rothe [47] are not shown as they are confined to a narrow range of energies not exceeding $0.04 \mathrm{Ry}$ above threshold (their data have been compared by Conneely et al. [31] and by Myerscough and Peach [48] to earlier calculations). Interestingly, the closecoupling calculations of Conneely et al. [31] lie closer to the $\mathrm{OPW}_{\mathrm{L}}$ results than they do to the $\mathrm{APW}_{\mathrm{L}}$, Robinson-Geltman, or Cooper-Martin results. The Moskvin PW calculations, not shown in Figure 12, predict a very large crosssection of almost $40 \mathrm{Mb}$ for $h \nu=0.34 \mathrm{Ry}$, but the $p \rightarrow s$ matrix elements are incorrect [48].

Tables IV and $\mathrm{V}$ list $\sigma$ values for $\mathrm{Br}^{-}$and $\mathrm{I}^{-}$, respectively. The energy in these tables is relative to that for the $J=3 / 2$ level, and is thus a measure of the kinetic energy only for the process producing that level. The OPW values in these tables differ from those in Tables III and IV by having the intensity distributed over the two $J$ levels and then summed for a given wavelength. We note that the APW values are larger than the $A P W_{L}$ values at energies below 0.2 Ry and agree better with both the $\mathrm{OPW}_{\mathrm{L}}$ values and the Robinson-Geltman results. As in previous cases, the $\mathrm{OPW}_{\mathrm{L}}$ results are lower at their maxima than are the other crosssections, but are larger at energies above 1 Ry. In general, the OPW decrease more slowly with energy than do the APW cross-sections, reflecting the inadequate treatment of the attractive potential by the former method. For $\mathrm{I}^{-}$, the 


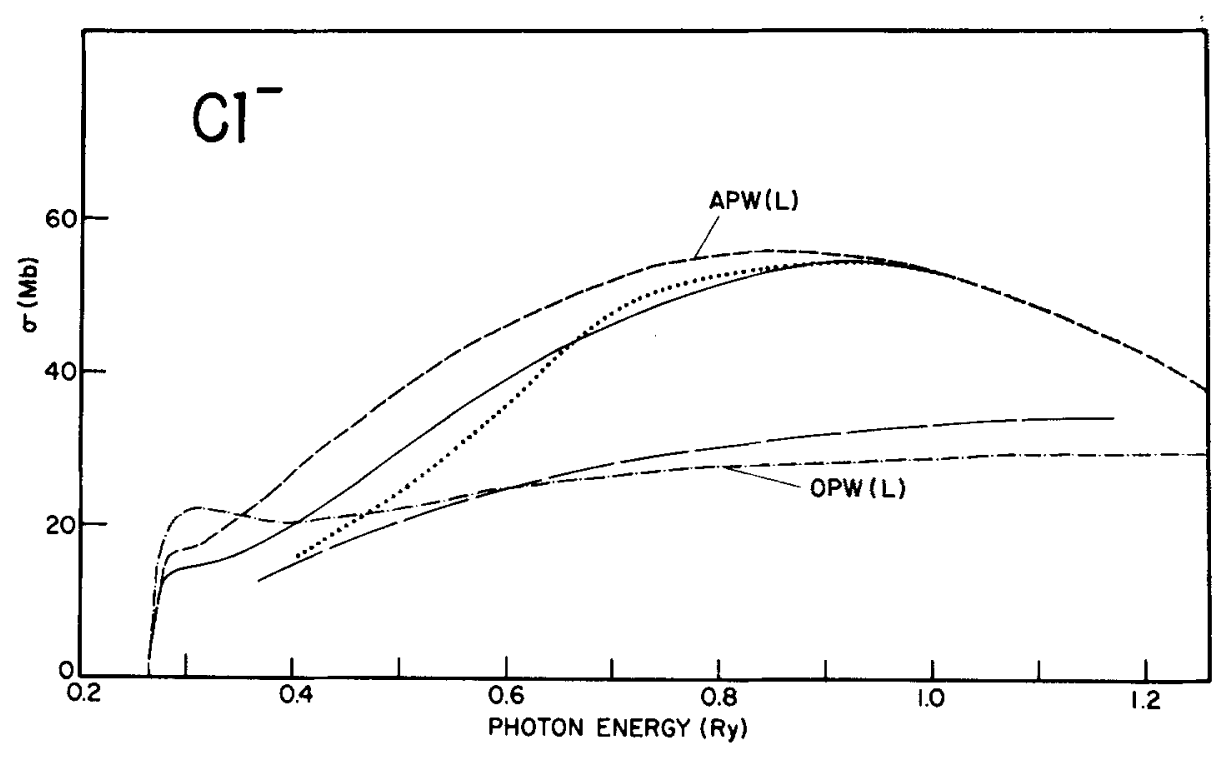

Figure 12. Cross-section $\sigma$ in $\mathrm{Mb}$ vs. photon energy in $\mathrm{Ry}$ for $\mathrm{Cl}^{-}$. APW (length) results with polarization denoted by (---) and OPW (length) results by (- - - ). Other results are those of Cooper and Martin [27] denoted by $(\cdots)$, Robinson and Geltman [28] by (-), and Conneely et al. [31] by (- - ). Energy shown as photon energy since all curves include the spin-orbit splitting of $0.008 \mathrm{Ry}$.

$\mathrm{OPW}_{\mathrm{L}}$ results in Figure 8 (without spin-orbit splitting) or Table V (with spin-orbit splitting) show a slight increase above $6 \mathrm{Ry}$, although the cross-section decreases at even higher energies.

In addition to the PW and OPW $\beta$ values described in Section 3B, Figures 5-8 display $\mathrm{APW}_{\mathrm{L}} \boldsymbol{\beta}$ values including core polarization. Values are not shown for energies below 0.2 Ry as they nearly coincide with the OPW curves. However, the $\mathrm{APW}_{\mathrm{L}}$ minima do occur at a lower energy than the PW or OPW minima; for example, for $\mathrm{Cl}^{-}$at approximately 0.07 Ry vs 0.18 Ry. As in the APW study [25] of inner $p$-shell photoionization, the dipole length and velocity $\beta$ values coincide within $2 \%$ or better except in regions where $\beta$ is varying rapidly with energy, so the velocity values are not shown. In the energy range from threshold to $3 \mathrm{Ry}$ the $A P W_{L} \beta$ values tend to be closer to the $O P W_{L}$ and $O P W_{V}$ values than to the $P W_{L}$ values. However, the second $\mathrm{APW}_{\mathrm{L}}$ minimum for $\mathrm{Cl}^{-}$in Figure 6 occurs near $2.9 \mathrm{Ry}$ rather than above $10 \mathrm{Ry}$ for the second $\mathrm{OPW}_{\mathrm{L}}$ minimum, probably as a result of the APW method's more nearly accurate treatment of the $s$ channel. The $\mathrm{APW}_{\mathrm{L}}$ curve for $\mathrm{Cl}^{-}$passes through zero at 2.7 and 3.1 $\mathrm{Ry}$, on either side of this second minima at $2.9 \mathrm{Ry}$. At $2.7 \mathrm{Ry}$ the $d$ channel matrix element $\boldsymbol{R}_{d}$ is zero, while at $3.1 \mathrm{Ry}$ the crossing results from $R_{d}=2 R_{s} \cos \Phi$ in Eq. (21), as does the lower energy crossing near $0.2 \mathrm{Ry}$. The $\mathrm{APW}_{\mathrm{L}} \beta$ values without polarization are similar, but with the second minimum shifted to $3.4 \mathrm{Ry}$. Although $\mathrm{APW}_{\mathrm{L}} \beta$ values 
TABLE IV. Photodetachment cross-sections (Mb) for $\mathrm{Br}^{--}$with polarization and spin-orbit coupling.

\begin{tabular}{|c|c|c|c|c|c|}
\hline$\lambda(\stackrel{D}{A})^{a}$ & $E(R y)$ & $\sigma_{L}(\mathrm{APW})$ & $\sigma_{V}(A P W)$ & $\sigma_{L}(O P W)^{b}$ & $\sigma_{L} \cdot\{R \& G)^{c}$ \\
\hline 3674 & 0.0 & 0.0 & 0.0 & 0.0 & 0.0 \\
\hline 3400 & 0.02 & 5.1 & 8.7 & 10.0 & 13.8 \\
\hline 3058 & 0.05 & 7.1 & 15.0 & 17.6 & 21.5 \\
\hline 2866 & 0.07 & 12.1 & 16.6 & 19.4 & 22.1 \\
\hline 2618 & 0.1 & 15.8 & 17.7 & 19.7 & 25.0 \\
\hline 2034 & 0.2 & 31.0 & 30.8 & 21.1 & 40.5 \\
\hline 1663 & 0.3 & 45.6 & 39.5 & 26.7 & 53.5 \\
\hline 1406 & 0.4 & 55.6 & 44.6 & 33.6 & 61.5 \\
\hline 1218 & 0.5 & 59.8 & 45.9 & 39.6 & 64.9 \\
\hline 1075 & 0.6 & 60.3 & 44.7 & 43.5 & 63.6 \\
\hline 961 & 0.7 & 57.9 & 41.3 & 45.8 & 58.2 \\
\hline 870 & 0.8 & 54.2 & 37.2 & 46.5 & 50.1 \\
\hline 794 & 0.9 & 49.0 & 33.4 & 45.9 & 41.1 \\
\hline 730 & 1.0 & 42.6 & 29.6 & 44.8 & $\longrightarrow$ \\
\hline 405 & 2.0 & 9.5 & 6.3 & 24.4 & $\ldots$ \\
\hline 214 & 4.0 & 0.83 & 1.0 & 5.9 & - \\
\hline 146 & 6.0 & 0.16 & 0.13 & 3.2 & - \\
\hline 110 & 8.0 & 0.11 & 0.10 & 0.8 & $\longrightarrow$ \\
\hline 89 & 10.0 & 0.08 & 0.08 & 0.8 & - \\
\hline
\end{tabular}

${ }^{\mathrm{a}} E A=0.248 \mathrm{Ry}, \Delta E_{\mathrm{so}}=0.034 \mathrm{Ry}$.

${ }^{\mathrm{b}}$ No polarization.

c Robinson and Geltman, Ref. [28].

for the other halides were not calculated for energies above $3 \mathrm{Ry}$, there is no zero value for $R_{d}$ for $\mathrm{F}^{-}$above threshold but below $10 \mathrm{Ry}$. For $\mathrm{Br}^{-}$and $\mathrm{I}^{-}$there are zero values of $R_{d}$ near 7 Ry with polarization and near 8 Ry without, so there must be at least one crossing in the same energy range as with the OPW results in Figures 7 and 8 . $^{*}$

\footnotetext{
* Note added in proof: Calculations of $\beta$ by the APW method have now been made for $\mathrm{F}^{-}, \mathrm{Br}^{-}$, and $\mathrm{I}^{-}$for electron kinetic energies from 3.0 to $10.0 \mathrm{Ry}$ in increments of $1.0 \mathrm{Ry}$, thus extending the curves denoted by (- $)$ in Figures 5, 7, and 8 . For $\mathrm{F}^{-}$the curve is nearly flat over this range and approaches a value of 1.49 at $10.0 \mathrm{Ry}$ in calculations including polarization and using the length operator. For $\mathrm{Br}^{-}$ there is minimum near $7.5 \mathrm{Ry}$, but $\beta$ is approximately 0.0 instead of -1.0 as in the $\mathrm{OPW}_{\mathrm{L}}$ and $\mathrm{OPW}_{\mathrm{V}}$ curves. For $\mathrm{I}^{-}$there is a similar shallow minimum in $\beta$ near $8.0 \mathrm{Ry}$. Thus, as already seen for $\mathrm{Cl}^{-}$in Figure 6, the APW calculations do not show the deep minima in $\beta$ in the range 1.0 to $10.0 \mathrm{Ry}$ that the OPW calculations show. Both methods do yield, however, deep minima below $1.0 \mathrm{Ry}$ with $\beta$ approaching $-1,0$. Qualitatively similar results are found in calculations without polarization.
} 


\section{Summary}

From the results and discussion in the previous section some conclusions may be drawn about the use of OPW continuum functions in describing photodetachment and photoionization processes:

1. The operator which should be used in connection with OPW continuum functions is the dipole length operator. The dipole velocity operator is not suitable in this case since the PW is an eigenfunction of this operator. A comparison of the

TABLE V. Photodetachment cross-sections $(\mathrm{Mb})$ for $\mathrm{I}^{-}$with polarization and spin-orbit coupling.

\begin{tabular}{|c|c|c|c|c|c|}
\hline$\lambda(\dot{A})^{a}$ & $E$ (Ry) & $\sigma_{L}(\mathrm{APW})$ & ${ }_{\mathrm{V}}(\mathrm{APW})$ & $\mathrm{c}^{(\mathrm{OPW})^{b}}$ & $\sigma_{L}(R \& G)^{c}$ \\
\hline 4032 & 0.0 & 0.0 & 0.0 & 0.0 & 0.0 \\
\hline 3861 & 0.01 & 1.8 & 9.3 & 10.5 & 16.6 \\
\hline 3302 & 0.05 & 3.9 & 13.8 & 15.5 & 17.5 \\
\hline 3079 & 0.07 & 5.5 & 15.9 & 16.2 & 27.0 \\
\hline 2978 & 0.08 & 7.6 & 20.6 & 21.4 & 30.3 \\
\hline 2795 & 0.1 & 10.9 & 24.6 & 25.2 & 37.2 \\
\hline 2139 & 0.2 & 32.2 & 41.4 & 28.6 & 69.0 \\
\hline 1732 & 0.3 & 59.0 & 54.8 & 38.4 & 92.8 \\
\hline 1456 & 0.4 & 82.0 & 60.8 & 51.1 & 97.7 \\
\hline 1255 & 0.5 & 91.8 & 59.5 & 62.7 & 89.0 \\
\hline 1103 & 0.6 & 91.4 & 53.0 & 70.4 & 73.3 \\
\hline 984 & 0.7 & 83.4 & 46.0 & 73.8 & 56.4 \\
\hline 888 & 0.8 & 70.7 & 38.9 & 73.1 & 41.6 \\
\hline 809 & 0.9 & 59.0 & 32.1 & 70.1 & 30.2 \\
\hline 743 & 1.0 & 48.2 & 25.7 & 62.0 & $\longrightarrow$ \\
\hline 409 & 2.0 & 6.4 & 4.2 & 19.3 & \\
\hline 216 & 4.0 & 0.78 & 0.49 & 1.6 & $\longrightarrow$ \\
\hline 146 & 6.0 & 0.20 & 0.15 & 1.2 & $\longrightarrow$ \\
\hline 111 & 8.0 & 0.10 & 0.09 & 3.1 & - \\
\hline 89 & 10.0 & 0.08 & 0.07 & 3.5 & - \\
\hline
\end{tabular}

${ }^{\mathrm{a}} E A=0.226 \mathrm{Ry}, \Delta E_{\mathrm{so}}=0.69 \mathrm{Ry}$.

${ }^{b}$ No polarization.

c Robinson and Geltman, Ref. [28].

$\mathrm{OPW}_{\mathrm{L}}$ and $\mathrm{OPW} \mathrm{V}_{V}$ cross-sections with the corresponding APW values, with other calculated values, and with experimental values, shows that there is a better agreement using the length operator. In addition, as pointed out by Reed et al. 
[19], only the length operator gives the correct threshold behavior for $p$-electron detachment into a PW final state. Most of the published PW and OPW results [1-11] for the photoionization of neutral molecules have, however, for reasons of convenience, been obtained exclusively with the velocity operator.

2. In OPW and PW methods the continuum states are essentially free waves. As such, they are better approximations for those photodetachment processes which result in continuum states with small phase shifts, namely states with high angular momentum. Therefore, one would expect that PW and OPW functions are poor for describing photodetachment from a $p$-orbital where one of the final states is an $s$ wave. As has also been frequently pointed out, these functions are better approximations to continuum states at high rather than low energies.

3. Although the $\mathrm{OPW}_{\mathrm{L}}$ values of both $\sigma$ and $\beta$ as a function of photon energy are in some instances in reasonable agreement with more reliable values, there is sufficient disagreement, even for atomic photodetachment, to suggest that indiscriminate use of the OPW method to describe photoionization of neutrals is unwarranted. In particular, we share the recent concern [37] about the significance of OPW $\beta$ values [10] for molecular photoionization.

It is obvious that the PW and OPW methods are far less accurate (at least at very low electron energies) than the APW and other theoretical methods discussed here, as they do not correctly account for the interaction between the ejected electron and the atomic core. However, the main attraction of the method is that it can be readily extended to molecular systems for which the other method are not easily applicable. Computationally, it is by far the cheapest method for crosssection calculations as all the integrals can be evaluated analytically.

The major advantage of the APW method* over the other related theoretical methods is that the APW method offers a more convenient and computationally cheaper means of obtaining continuum wave-functions. Our formulation of the method, however, does not explicitly include polarization and exchange potentials. In all of the other methods reviewed here, at least the continuum function and sometimes both the bound and continuum functions, are obtained by the numerical integration of the corresponding radial wave equation. In the APW method, however, the solutions of the radial equation for each region of the potential are known. The continuum-state solution for the entire range of potential is constructed from these "regional" solutions, smoothly joined together by the application of suitable boundary conditions.

\section{Acknowledgment}

The authors wish to thank the University of Michigan Computing Center for the use of its facilities. One of us (L.L.L.) would like to thank the Department of Chemistry of the University of California, Berkeley, for its hospitality during the writing of this paper.

\footnotetext{
* The term APW is usually used for a related method for solids involving numerical integrations within a spherical region and including exchange via a statistical approximation [49].
} 


\section{Bibliography}

[1] I. G. Kaplan and A. P. Markin, Opt. Spectrosc. (U.S.A.) 24, 475 (1968); 25, 275 (1968) [Opt. Spektrosk. (USSR) 24, 884 (1968); 25, 493 (1968)].

[2] I. G. Kaplan and A. P. Markin, Sov. Phys. Dokl. (U.S.A.) 14, 36 (1969) [Dokl. Akad. Nauk SSSR 184, 66 (1969)].

[3] L. L. Lohr, Jr. and M. B. Robin, J. Amer. Chem. Soc. 92, 7241 (1970).

[4] L. L. Lohr, Jr., Electron Spectroscopy: Proceedings of the International Conference on Electron Spectroscopy, Asilomar, Pacific Grove, Calif., Sept. 1971, D. A. Shirley, Ed. (North-Holland, Amsterdam, 1972), pp. 259-267.

[5] W. Thiel and A. Schweig, Chem. Phys. Lett. 12, 49 (1971); 16, 409 (1972); 21, 541 (1973).

[6] A. Schweig and W. Thiel, J. Chem. Phys. 60, 951 (1974).

[7] J.-T. J. Huang, F. O. Ellison, and J. W. Rabalais, J. Electron Spectrosc. 3, 399 (1974).

[8] J.-T. J. Huang and F. O. Ellison, J. Electron Spectrosc. 4, 233 (1974).

[9] F. O. Ellison, J. Chem. Phys. 61, 507 (1974).

[10] J. W. Rabalais, T. P. Debies, J. L. Berkosky, J.-T. J. Huang, and F. O. Ellison, J. Chem. Phys. 61, $516,529(1974)$.

[11] T. P. Debies and J. W. Rabalais, J. Amer. Chem. Soc. 97, 487 (1975).

[12] For example, see S. Iwata and S. Nagakura, Mol Phys. 27, 425 (1974).

[13] For $\mathrm{OH}^{-}$, see L. M. Branscomb, Phys. Rev. 148, 11 (1966); for $\mathrm{SH}^{-}$, see B. Steiner, J. Chem. Phys. 49, 5097 (1968).

[14] For $\mathrm{PH}_{2}^{-}, \mathrm{NH}_{2}^{-}$, $\mathrm{AsH}_{2}^{-}$, and $\mathrm{SH}^{-}$, see K. C. Smyth and J. I. Brauman, J. Chem. Phys. 56, 1132, 4620, 5993 (1972).

[15] D. R. Bates and H. S. W. Massey, Astrophys. J. 91, 202 (1940).

[16] S. Chandraskhar, Astrophys. J. 102, 223 (1945).

[17] Yu. V. Moskvin, Opt. Spectrosc. (U.S.A.) 14, 270 (1964) [Opt. Spektrosk. (USSR) 17, 499 (1964)].

[18] Yu. V. Moskvin, High Temp. (U.S.A.) 3, 765 (1965) [Teplofiz. Vysokikh Temp. (USSR) 3, 821 (1965)].

[19] K. J. Reed, A. H. Zimmerman, H. C. Andersen, and J. I. Brauman, J. Chem. Phys. 64, 1368 (1976).

[20] L. C. Allen, J. Chem. Phys. 34, 1156 (1961); for corrections, see L. L. Lohr, Jr. and L. C. Allen, J. Chem. Phys. 39, 2106 (1963).

[21] R. E. Watson and A. J. Freeman, Phys. Rev. 123, 521 (1961).

[22] E. Clementi, "Tables of Atomic Functions," IBM J. Res. Develop., unpublished supplement (1965).

[23] M. Synek and P. Grossgut, Phys. Rev. A5, 2684 (1972).

[24] E. J. McGuire, Phys. Rev. 161, 51 (1967); 175, 20 (1968). Also see Phys. Rev. A3, 267 (1971) for applications to inelastic electron and proton scattering.

[25] F. M. Chapman, Jr. and L. L. Lohr, Jr. J. Amer. Chem. Soc. 96, 4731 (1974). Also see F. M. Chapman, Jr., Ph.D. dissertation, The University of Michigan, Ann Arbor, Michigan, 1973.

[26] J. W. Cooper and R. N. Zare, Lectures in Theoretical Physics, Vol. 11C, S. Galtman, K. Mahanthappa, and W. Brittin, Eds., (Gordon and Breach, New York, 1969), pp. 317-337.

[27] J. W. Cooper and J. B. Martin, Phys. Rev. 126, 1482 (1962).

[28] E. J. Robinson and S. Geltman, Phys. Rev. 153, 4 (1967).

[29] F. Herman and S. Skillman, Atomic Structure Calculations (Prentice-Hall, Englewood Cliffs, N.J., 1963).

[30] W. R. Garrett and H. T. Jackson, Jr., Phys. Rev. 153, 28 (1967).

[31] M. J. Conneely, K. Smith, and L. Lipsky, J. Phys. B3, 493 (1970).

[32] T. Ishihara and T. C. Foster, Phys. Rev. A9, 2350 (1974).

[33] R. S. Berry and C. W. Reimann, J. Chem. Phys. 38, 1540 (1963). Also see the reviews by B. M. Smirnov, High Temp. (U.S.A.) 3, 716 (1965) [Teplofiz. Vysokikh Temp. (USSR) 3, 775 (1965)] and B. Steiner, Case Studies in Atomic Collision Physics, Vol. II, E. W. McDaniel and M. R. C. McDowell, Eds. (North-Holland, Amsterdam, 1972), pp. 483-545. 
[34] E. P. Wigner, Phys. Rev. 73, 1002 (1948).

[35] J. W. Cooper and S. T. Manson, Phys. Rev. 177, 157 (1969).

[36] D. J. Kennedy and S. T. Manson, Phys. Rev. A5, 227 (1972).

[37] J. S. Shyu and S. T. Manson, Phys. Rev. A11, 166 (1975).

[38] J. Cooper and R. N. Zare, J. Chem. Phys. 48, 942 (1968); 49, 4252 (1968).

[39] J. L. Hall and M. W. Siegel, J. Chem. Phys. 48, 943 (1968).

[40] A. F. Starace, Phys. Rev. A3, 1242 (1971); A8, 1141 (1973).

[41] M. Cohen and R. P. McEachran, Chem. Phys. Lett. 14, 201 (1972).

[42] A. Dalgarno, Advan. Phys. 11, 281 (1962).

[43] A. Mandl, Phys. Rev. A3, 251 (1971).

[44] H.-P. Popp, Z. Naturforsch. 22a, 254 (1967).

[45] C. E. Moore, "Atomic Energy Levels" USNBS Circular No. 467, Vol. I (1949), Vol. II (1952), Vol. III (1958).

[46] G. Muck and H.-P. Popp, Z. Naturforsch. 23a, 1213 (1968).

[47] D. E. Rothe, Phys. Rev. 177, 93 (1969).

[48] V. P. Myerscough and G. Peach, Case Studies in Atomic Collision Physics, Vol. II, E. W. McDaniel and M. R. C. McDowell, Eds. (North-Holland, Amsterdam, 1972), pp. 293-397.

[49] J. C. Slater, Quantum Theory of Molecules and Solids, Vol. 2 (McGraw-Hill, New York, 1965), pp. 233-236 and references therein.

Received August 6, 1975 